\section{Tuberculosis contact tracing: are the British Thoracic Society guidelines still appropriate?}

S F Hussain, R Watura, Beryl Cashman, I A Campbell, M R Evans

\begin{abstract}
The value of contact tracing in South Glamorgan for the period May 1987 to December 1989 was assessed. Tuberculosis was diagnosed in six (3.5\%) of 170 close contacts. All were young and were detected in the initial screening. Tuberculosis was not found among 441 casual contacts. The value of follow up of contacts after the initial screening and of screening casual contacts in districts with a low prevalence of tuberculosis is questioned.
\end{abstract}

(Thorax 1992;47:984-985)

The British Thoracic Society guidelines recommend periodic radiological examination of unvaccinated close contacts of smear positive index cases who have grade 2 Heaf test reactions and all Heaf grade 3 and 4 close contacts for up to two years in Indian Subcontinent contacts and for one year in contacts not originating from the Indian Subcontinent.' Most disease in contacts is found at the initial examination and the value of contact tracing beyond six months has been questioned. ${ }^{2} \mathrm{We}$ assessed the value of contact tracing in South Glamorgan (population about 400000 with $1 \cdot 2 \%$ from the Indian Subcontinent) from May 1987 to the end of December 1989.

Medicine, Llandough Hospital, Penarth, Cardiff CF6 1XX

S F Hussain

B Cashman

I A Campbell

Centre for Applied

Public Health and Department of Public

Health Medicine,

Temple of Peace and

Health, Cardiff

CF1 3NW

R Watura

M R Evans

Reprint requests to:

Dr I A Campbell

Received 6 November 1991

Returned to authors

24 January 1992

Revised version received

24 February 1992

Accepted 11 March 1992

\section{Patients and methods}

All patients with tuberculosis notified from May 1987 to December 1989 in South Glamorgan were identified and classified into two ethnic groups-Indian Subcontinent (ISC) and non-ISC. The site of the tuberculosis was recorded as pulmonary or nonpulmonary. Contacts were classified as close or casual. Close contacts were defined as members of the same household as the notified case who shared kitchen or bathroom facilities or who were very close associates-for example, relatives who visited frequently and girl or boy friends. All other contacts were called casual.
The contact tracing procedure was based on the British Thoracic Society guidelines. ${ }^{3}$

Data were collected mainly from records in the contact tracing clinic and additional bacteriological information was obtained from the Mycobacterium Reference Unit, Cardiff.

Results were analysed by means of the Survey Plus and Epi-info computer packages. $\chi^{2}$ tests were performed with Yates's correction.

\section{Results}

One hundred and one index cases were identified, 22 of them of ISC ethnic origin. The mean ages were $37 \cdot 6$ (SE 3.4) years for the ISC cases and 55.3 (SE 2.2) years for the others ( $p<$ $0.01)$. The sex distribution in the two groups was not significantly different (M:F 10:12 for ISC, $44: 35$ for non ISC).

Seventy one $(70 \cdot 3 \%)$ index cases had pulmonary tuberculosis (16 ISC and 55 non-ISC) and $30(29 \cdot 7 \%)$ non-pulmonary (six ISC and 24 non-ISC). The differences between the ISC and non-ISC groups in site of tuberculosis, smear positivity (5/16 $v 31 / 55)$, and culture positivity $(10 / 22 v 51 / 79)$ were not statistically significant.

Six hundred and eleven contacts were identified. The mean ages were 20.4 (SE 1.4) year for ISC cases and 34.7 (SE 1.1) years for nonISC cases $(p<0.001)$. The table summarises the results of the initial contact screening.

Tuberculosis was diagnosed in six (three ISC, three non-ISC) contacts, a yield of $1 \%$ from all contacts or $3.5 \%$ from close contacts. All were detected by the initial screening, five through contact tracing (one was already in hospital). All were below 26 years of age and four were under 10. All were close contacts and none were known to have had BCG vaccination. The index cases were smear positive for five of these contacts; one index case was smear negative but culture positive. No contact of any patient with culture negative pulmonary or non-pulmonary tuberculosis developed disease. The average delay between notification and screening was two to four weeks, being less than 31 days in all contacts diagnosed as having tuberculosis. Chemoprophylaxis was given to a further four contacts who had Heaf grade 2-4 reactions and in whom tuberculosis was no diagnosed. All were close contacts of index cases with smear positive pulmonary disease and none had had BCG vaccination.

Twenty eight $(21.4 \%)$ ISC and $42(9.0 \%)$ 'non-ISC contacts were given follow up appointments, most being close contacts of index cases with pulmonary disease. The overall attendance rates at six, 12 and 24 months were $83 \%$, $36 \%$, and $30 \%$ respectively. No new case of tuberculosis was diagnosed at these follow ups. No contact is due for any further follow up visit, and no new case has been notified in South 
Details of initial screening of contacts of Indian Subcontinent (ISC) and non-ISC cases

\begin{tabular}{|c|c|c|c|}
\hline & \multicolumn{2}{|c|}{ No $(\%)$ of contacts } & \multirow[b]{2}{*}{$p$} \\
\hline & $\begin{array}{l}\text { ISC } \\
\text { ( } 22 \text { index cases) }\end{array}$ & $\begin{array}{l}\text { Non-ISC } \\
\text { ( } 79 \text { index cases) }\end{array}$ & \\
\hline Total No of contacts & 133 & 478 & \\
\hline Close & $82(61 \cdot 7)$ & $88(18 \cdot 4)$ & $<0.001$ \\
\hline Casual & $51(38 \cdot 3)$ & $390(81 \cdot 6)$ & $<0.001$ \\
\hline No screened & $131(98 \cdot 5)$ & $466(97 \cdot 5)$ & \\
\hline BCG history & $32(24 \cdot 4)$ & $82(17 \cdot 6)$ & \\
\hline Heaf test & $89(67 \cdot 9)$ & $243(52 \cdot 1)$ & \\
\hline negative & $38(42 \cdot 7)$ & $141(58 \cdot 0)$ & $<0.05$ \\
\hline $\begin{array}{l}\text { Tuberculosis diagnosed in } \\
\text { contacts }\end{array}$ & $3(2 \cdot 3)^{\star}$ & $3(0 \cdot 6)^{\star}$ & \\
\hline
\end{tabular}

^All were close contacts. diagnosis of tuberculosis in the contacts was made in the initial screening. No casual contact or any contact of non-pulmonary tuberculosis developed disease. In our district (tuberculosis notification rate 40 a year) we question the value of following up contacts after the initial screening and the value of screening casual contacts at all. The contact tracing guidelines in the British Thoracic Society "updated code of practice" ' are derived from data gathered in the 1970 s. $^{5}$ Our results, taken with those from Leeds ${ }^{6}$ and Blackburn, ${ }^{7}$ suggest that consideration of regional demographic differences are important for the development of guidelines that are cost effective and more appropriate to Britain in the 1990s. Perhaps the time has come for a new nationwide study.

We thank Dr P A Jenkins, director, Mycobacterium Reference Unit, Cardiff, and Dr D J Evans, senior registrar in dental public health, South Glamorgan Health Authority, for their help and health,
advice.

Glamorgan among those who were discha after initial screening or who defaulted from follow up.

\section{Discussion}

There are about 5000 new notifications of tuberculosis a year in the United Kingdom. ${ }^{4}$ Contact tracing is essential for the detection of asymptomatic cases of tuberculosis but is expensive and time consuming. Our results support the view that those at greatest risk of developing tuberculosis are young and in close contact, without previous BCG vaccination, where the index case had smear positive pulmonary tuberculosis. ${ }^{5}$ Without exception the
1 Subcommittee of the Joint Tuberculosis Committee of the British Thoracic Society. Control and prevention of tuberculosis in Britain: an updated code of practice. $B M J$ 1990;300:995-9.

2 Selby CD, Allen MB, Leitch AG. Optimal duration of follow-up for tuberculosis contacts. Respir Med 1989; 83:353-5.

3 Joint Tuberculosis Committee. Control and prevention of cuberculosis: a code of practice. $B M J$ 1983;287:1118-21.

4 Office of Population Censuses and Surveys. Communicable disease statistics. Series MB2. London: HMSO, 1991.

5 British Thoracic Association. A study of standardised contact procedures in tuberculosis. Tubercle 1978;59:245-9.

6 Teale C, Cundall DB, Pearson SB. Time of development of tuberculosis in contacts. Respir Med 1991;85:475-7.

7 Ormerod LP. Results of tuberculosis contact tracing: Blackburn 1982-90 [abstract]. Thorax 1991;46:35P. 